\title{
The presence of multimedia in astronomy teaching
}

\author{
By H. J. Fogh Olsen \\ Copenhagen University Observatory, Denmark
}

\section{Introduction}

Sometimes I find my self in a society in the middle of The Global Village and sometimes in a society in a little state with a large number of computers not speaking the language I usually talk. When a prevailing part of the population are working in one area the society is named after that area, allthough a lot of other things can characterize the society. The latest societies are:

- Agricultural society

- Industrial society

- Information society

The agricultural and industrial societies have come to an end. When a society comes to an end, it is usually because the efficiency of production reaches a level higher than necessary, to keep all the workers busy. Many of the workers are attracted to other kinds of work, which gives rise to the next culture.

We must imagine a similar over production of information, so that the number of people occupied by producing information will start to decline. Some say that we have reach the end point already, because we have access to information from all over the world through computers, Internet and World Wide Web in an amount larger than we can handle. But that may not be true because we are waiting for large numbers of the population to learn to utilize all that information. The demand may increase for some time to come.

We can see the extremly high impact computers have on politicians, compared to their previous interest in libraries. In Denmark more money has been put into school computers during the last five years, than have ever been used for school library books.

\section{Media carrying information}

Up to a few decades ago it was difficult for the general public to get substantial information about more complicated subjects such as science. Although the books have existed for quite a long time they were not for the general public but only for people with more academic interest.The period starting with Isaac Newton, can be described by words and mathematics, and so it has been for 300 years. But it has also been dominated by demonstrations of the physical laws. Many sophisticated models demonstrating the physical laws at work among our planets have been produced over the years.

\subsection{Planetary models from Ole Romer.}

The Jovilabium made by Ole Romer demonstrates how the moons of Jupiter can orbit Jupiter and look the same as seen in a small telescope from Earth. The Eclipsarium demonstrates when either a lunar or a solar eclipse will occur. Only a few solar eclipses from the polar regions are not "discovered"by the machine. The remaining eclipses are all included at their precise time of appearance.

The planetarium, modelling all the planetary orbits, was also produced by Ole Romer. Several examples of a royal transportable model were made in Paris, and a more public model was made for The Round Tower in Copenhagen. All were hand driven. The latter now exists as a copy in Tycho Brahe Planetarium in Copenhagen. The other models made by Ole Romer exist in copies at the Ole Romer museum in Copenhagen. Since the first utilisation of electricity, a projection planetarium was soon introduced and has developed during the following 100 years to the point where we now have CRT projectors. 


\subsection{Graphics and Images}

There is no doubt that the human brain is very efficient, when it receives impressions from the eyes either directly from nature or from images, graphics or drawings. Images are very valuable, but have not been used much in physics. Images from the real world are compared with nature, so the images is always related in size, colour etc.

Astronomical images made with telescopes differ from terrestrial telescope images, in the sense that you can not make a comparison of image and reality, because we cannot perceive the Universe with the naked eye.

The disappointment of observing a star through a telescope has happened to almost everybody who has the chance to do so, because the star looks the same and does not show the detail expected. The beautiful images from the sky are not available to the unaided eye with or without a telescope. They must therefore be explained in more detail than would be the case for a terrestrial image. Not many teachers in Denmark have introduced the use of astronomical images.

\subsubsection{Globular Clusters}

Graphic representation is so much easier, but should also be taken with care.

A classical example is the presentation of The Milky Way Galaxy. The Globular Clusters are often shown distributed uniformly around a line, occupying a rather large area. The Globular Clusters are located far from the Galactic Plane and the Central Bulge, which is not the reality. Does such display add anything to understanding of the structure of the Galaxy?

2.2.2 The $H$ - $R$ diagram.

If you try to find

- The location of the Sun

- The luminosity of white dwarfs

- The lowest effective temperature of a star

you may reach very different results. In two pages of a text book you must learn to understand why astronomers use absolute magnitude, luminosity, apparent magnitude or spectral type, effective temperature or colour to show the same linear relation but with the plotted line placed in a funny way because astronomers use opposite signs for the axes to normal.

\subsubsection{The Moon}

The last example of that sort is also a classical one. Since it was very expensive to produce illustrations for books, it was an advantage to put as much information as possible into one illustration as possible. When you want to illustrate the phases of the Moon astronomers are able to do that in one figure. From what point on earth would you be able to observe the moon as illustrated?

Who can blame the illustration of the more popular literature when they play with images to give a more fascinating background. But how is it possible for the innocent reader to distinguish between that and a scientific image produced to describe new scientific areas?

\section{Cyberspace and virtual reality in a digitized culture.}

- teachers' explanations and blackboard work

- prose and mathematical formulas

- graphics and images

- models and experiments

Taking up a new name like multimedia, it can be hard to imagine what it should be. After all it is nothing new, except that it is just everything from everywhere brought together at a time you wish from The Global Village.

All these media have been digitized for the purpose of being electronically handled by wire from the storage medium to the CRT. The storage medium is usually a hard disc or a CD-ROM but can be one of the many other systems in use today or new systems to be developed in the future. One advantage and important thing is the way it has been established almost free of charge and available to an overwhelming and rapidly growing number of people.

One disadvantage is the extremly fast way it changes so what you may have found interesting 
one day is not available the next and it happens to be published in no more than a single copy. Very often you only know the date it appeared, you do not know who wrote it or published it. Sometimes you may find the previous text etc. but it does not look the same. You cannot really rely on it for reference. Since you receive information from all over the world and from all levels of sources it is difficult even for professionals to judge the value of the data received. School pupils value is crucial because the most exciting data is often not appropriate for teaching.

The resulting consequence of this new access to information is already with us; in the future it will be more complicated to teach but maybe easier to learn.

In the old simplified model the teacher is able to control all information. The teacher selects the books to be utilized.

In Denmark, you cannot find any books describing science in the Danish language other than the teachers choice of text book. Books in foreign language are usually not available and certainly not at the high school level or popular science level.

Up to now the teacher keeps control of the curriculum since the pupils are forced to follow the set guidelines. In the near future everybody will have access to The World Wide Web and they will be able to collect information of all kinds and levels in many languages. Teachers need to change their job from carrying information to the pupils-trainers, or maybe word coachs will be more appropriate in the future.

Probably the most obvious thing is to start at university level but very soon Internet connection will be available to many schools too. We should not hesitate to take the initiative to set up schemes to evaluate the quality and value of information on the Internet. How can we ensure that the information obtained from the Internet is of the necessary quality? At least in Denmark, the astronomical or probably physical curriculum must include some new items:

- evaluation of source material

- testing individual students progress

- recording the transient Internet sources

- reviews of www information

It is not the time to find a standard, but it is the time to discuss how we ensure that the previous high standard of information at school level is maintained when information of all kinds of quality continually arrives from the World Wide Web. 